\title{
Lensless on-chip coded aperture imaging using multi-angle LEDs (Withdrawal Notice)
}

Atif Hassan, Sohail Khan, Khurram Rasul, Anwar Hussain

Atif Hassan, Sohail Khan, Khurram Rasul, Anwar Hussain, "Lensless on-chip coded aperture imaging using multi-angle LEDs (Withdrawal Notice)," Proc. SPIE 11549, Advanced Optical Imaging Technologies III, 115490W (4 November 2020); doi: 10.1117/12.2572697

SPIE. Event: SPIE/COS Photonics Asia, 2020, Online Only 


\section{Lensless on-chip coded aperture imaging using multi-angle LEDs (Withdrawal Notice)}

\section{Atif Hassan, Sohail Khan, Khurram Rasul, Anwar Hussain COMSATS University Islamabad (Pakistan)}

Proceedings Volume 11549, Advanced Optical Imaging Technologies III, 115490W (10 October 2020) https://doi.org/10.1117/12.2572697

Online Publication Date: 10 October 2020

Withdrawn from Publication: 28 October 2020

Event: SPIE/COS Photonics Asia Digital Forum 2020

Conference Date: 11-16 October 2020

This presentation, originally published on 10 October 2020, was withdrawn on 28 October 2020 per author request. 